\title{
ASPECTOS IMUNOLÓGICOS DA HANSENÍASE
}

\author{
IMMUNOLOGICAL ASPECTS OF LEPROSY
}

Norma Tiraboschi Foss

Docente do Departamento de Clínica Médica da Faculdade de Medicina de Ribeirão Preto da Universidade de São Paulo.

CorRESPOndÊnCIA: Norma Tiraboschi Foss - Divisão de Dermatologia - Departamento de Clínica Médica da Faculdade de Medicina de Ribeirão Preto - Campus Universitário - CEP: 14048-900 - Ribeirão Preto - SP.

FOSS NT. Aspectos imunológicos da hanseníase. Medicina, Ribeirão Preto, 30: 335-339, jul./set. 1997.

RESUMO: A distribuição espectral das formas clínicas da hanseníase tem sido associada a alterações imunológicas do hospedeiro, frente ao $M$. leprae. Foi verificado que, no pólo de maior suscetibilidade, hanseníase virchoviana, apresenta depressão da resposta imune celular, associada ao aumento da produção de citocinas supressoras (IL-4 e TGF $\beta$ ) e redução dos níveis de citocinas ativadoras da resposta imune (TNF $\alpha$, IL-1 e IFN $\gamma$ ). Há, ainda, menor produção de elementos responsáveis pela oxidação intracelular, como intermediários do nitrogênio (RNI), avaliada pela expressão da enzima NO sintase induzida (iNOS). Por outro lado, no pólo de resistência, hanseníase tuberculóide, não foram detectadas as citocinas supressoras, em contraste com elevadas concentrações de TNF $\alpha$ e IL-1, citocinas indutoras da reação inflamatória.

UNITERMOS: Hanseníase; imunologia.

\section{ESPECTRO CLÍNICO-IMUNOPATOLÓ- GICO DA HANSENÍASE}

Sendo a hanseníase doença infecto-contagiosa, que se caracteriza por apresentar formas clínicas, patológicas e imunológicas, contrastantes, o seu estudo imunopatológico torna-se fundamental para o entendimento dos mecanismos fisiopatológicos, envolvidos no desenvolvimento da doença.

Baseando-se na história natural da doença, observa-se que existe uma forma de alta resistência à infecção pelo Mycobacterium leprae, a hanseníase tuberculóide (HT), na qual as manifestações estão relacionadas à exacerbação da resposta imune celular, ocorrendo formação de granuloma bem definido, limitação das lesões e destruição completa dos bacilos. No outro pólo, encontra-se a forma de alta suscetibilidade, a hanseníase virchoviana (HV), que se caracteriza por deficiência de resposta imune celular, excessiva multiplicação bacilar e disseminação da infecção para vísceras e tecido nervoso. É a forma de importância epidemiológica, pois os bacilos estão maciçamente presentes nas lesões cutâneas, na proporção de $10^{10}$ bacilos/g tecido ${ }^{1}$, propiciando a transmissão da doença por contato físico.

Entre estas formas polares, ilustradas na Figura 1, situam-se as formas instáveis da doença, com amplo espectro de manifestações clínicas. A hanseníase dimorfa $($ HD), pode adquirir características tuberculóide (HDT) ou virchoviana (HDV), ou, simplesmente, permanecer como dimorfa (HDD), dependendo da potencialidade de resposta imune celular do hospedeiro parasita.

A medida da resposta imunológica pode ser efetuada através da reação de Mitsuda, que consiste na inoculação intradérmica de suspensão de bacilos (humanos ou provenientes do tatu) mortos pelo calor, cuja leitura é feita após 04 (quatro) semanas, resultando em pápula infiltrada (Reação Positiva) ou ausência de alteração cutânea (Reação Negativa). 


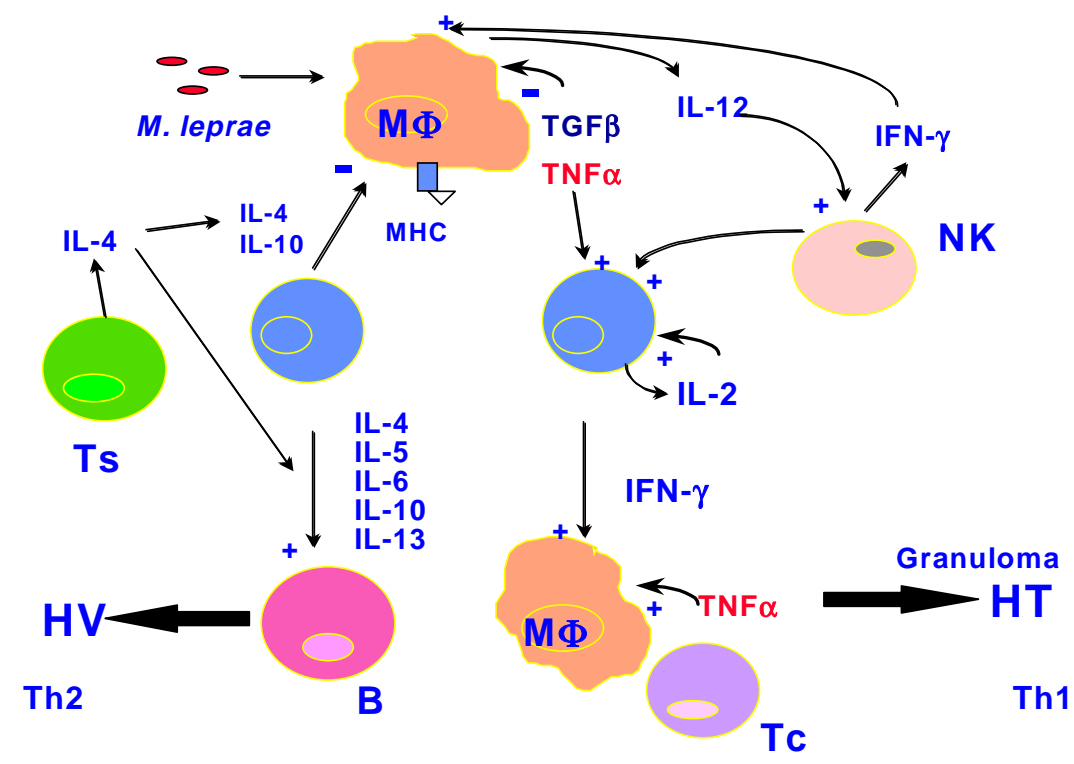

Figura 1 - Imunologia da hanseníase.

$M \Phi=$ macrófago; $N K=$ célula NK - natural killer; $\mathrm{Th}_{1}-\mathrm{Th}_{2}=$ subpopulações de linfócito $\mathrm{T}-\mathrm{CD}_{4}{ }^{+}$;

$\mathrm{B}=$ linfócito $\mathrm{B} ; \mathrm{Tc}=$ linfócito $\mathrm{T}$ citotóxico; $\mathrm{Ts}=$ linfócito $\mathrm{T}$ supressor; $\mathrm{IL}_{1} \ldots \mathrm{IL}_{12}=$ interleucinas $1 \ldots 12$;

TNF $\alpha=$ fator de necrose tumoral; IFN $\gamma=$ interferon $\gamma$; TGF $\beta=$ fator transformador do crescimento $-\beta ; \mathrm{HT}=$ hanseníase tuberculóide; $\mathrm{HV}=$ hanseníase virchoviana.

Segundo o resultado da reação de Mitsuda, os pacientes podem ser classificados em suscetíveis ou resistentes à doença, pois a reação é sempre negativa em virchovianos e positiva em tuberculóides.

Tem sido observado que virchovianos e borderline-virchovianos cursam, em sua evolução, com elevadas concentrações de anticorpos no soro, especialmente os anticorpos específicos do M.leprae, como o anti-PGL1, associado com a depressão da imunidade celular.

O PGL1, glicolipídeo fenólico-1, componente de parede celular, específico do $M$. leprae, que constitui cerca de $2 \%$ da massa total bacteriana, caracteriza-se por ser um antígeno específico do bacilo, podendo ser encontrado em tecidos, no sangue circulante e na urina de doentes multibacilares. Foi descrita sua estrutura, composta de um trissacarídeo, fenol, fitiocerol e de ácido micoseossídico ${ }^{2,3,4}$. É detectado também em tatus infectados com o M. leprae ${ }^{2,5,6}$. Este antígeno não apresenta reação cruzada com M. tuberculosis ou outras microbactérias.

$\mathrm{Na}$ hanseníase virchoviana, apesar da exacerbação e especificidade da resposta humoral, esta é inefetiva para a eliminação dos bacilos. A eficácia da defesa é efetuada por células capazes de fagocitar a bactéria e destruí-la (células apresentadoras de antígenos - macrófagos e outras), apresentando às células $\mathrm{T}$ auxiliares apenas a sua fração antigênica, em associação com complexo de histocompatibilidade principal (MHC). Nesta etapa, há interação entre o macrófago e os linfócitos $\mathrm{T}$, capazes de reconhecer o antígeno através de receptores de superfície, desencadeando a resposta imune celular. Esta resposta é mediada por diversos fatores, entre eles, as interleucinas ou citocinas, que são glicopeptídeos relacionados às reações intercelulares.

Macrófagos produzem as citocinas, IL-1, $\alpha$ TNF, IL-6 e IL-12, que atuam sobre linfócitos T, geralmente a população de fenótipo CD4+ (helper ou auxiliares), que passam a produzir suas próprias citocinas. A IL-12 estimula diretamente a célula NK, induzindo a produção de IFN- $\gamma$, com função de potencializar a ativação do macrófago.

\subsection{Resposta imunológica Th1 e Th2}

Linfócitos CD4+ são subdivididos em Th1 e Th2, com atividades imunorreguladoras específicas, que são mediadas pelas citocinas. A subpopulação Th1 
produz as citocinas IL-2, IFN- $\gamma$ e TNF- $\beta$, responsáveis pela manutenção da resposta imune celular. A IL-2 ativa receptores dos linfócitos CD4+, estimulando a formação de clones celulares, responsáveis pela manutenção da produção de citocinas e, paralelamente, estimulam células NK, com ação de potencializar uma maior produção de IFN- $\gamma$.

O IFN- $\gamma$ age sobre macrófagos, estimulando a fagocitose e os mecanismos de ativação celular, levando a maior produção de TNF- $\alpha$, que incrementa a ativação macrofágica, e atuando através de um mecanismo sinérgico cíclico.

A subpopulação Th2 produz as citocinas IL-4, IL-5, IL-6, IL-8 e IL-10. IL-4 e IL-10, que são supressoras da atividade macrofágica, produzindo bloqueio da estimulação de macrófagos, com conseqüente desvio da resposta imunológica. Adicionalmente, IL-4 estimula linfócitos B, que se tornam produtores de imunoglobinas, e mastócitos, que passam a produzir mais IL-4, incrementando a resposta supressora macrofágica.

Assim, dependendo da subpopulação de células $\mathrm{T}$ em atividade, durante o processo inflamatório, haverá predominância de mecanismos de defesa ou de disseminação da doença (Figura 1).

Defesa e/ou resistência à infecção estão relacionadas à presença de TNF- $\alpha$ e à produção de mediadores de oxidação, como reativos intermediários do oxigênio (ROI) e do nitrogênio (RNI), elementos fundamentais para a destruição bacilar, intramacrofágica ${ }^{7,8}$.

Entretanto, o M. leprae pode apresentar mecanismos de escape à oxidação intramacrofágica, pela produção dos antígenos PGL1 e LAM (lipoarabinomanana), com função supressora da ativação de macrófagos, proporcionando condições para que o bacilo fique protegido no citoplasma desta célula, multiplicando-se e formando globias (Células de Virchow).

\section{CORRELAÇÃO ENTRE CITOCINAS E MANIFES- TAÇÕES CLÍNICAS}

Além dos quadros clínicos característicos, doentes virchovianos apresentam, durante o tratamento específico, surtos reacionais, caracterizados por nódulos eritematosos, febre, astenia, artralgia e outros achados típicos de uma reação inflamatória aguda. O envolvimento destes fatores, com a fisiopatologia da hanseníase, tem sido objeto de nossos estudos nos últimos anos.

Em estudo pioneiro para a detecção da citocina TNF- $\alpha$ na hanseníase, foi observado que esta citocina está presente no soro de doentes tuberculóides e ausente em soro de virchovianos, e que estes resultados estavam correlacionados com a produção de TNF- $\alpha$ por células mononucleares, de doentes com as formas polares da doença, indicando que a destruição do M. leprae e a formação de granuloma estão associadas à presença de TNF- $\alpha$, como mostra a Figura $2^{9}$.

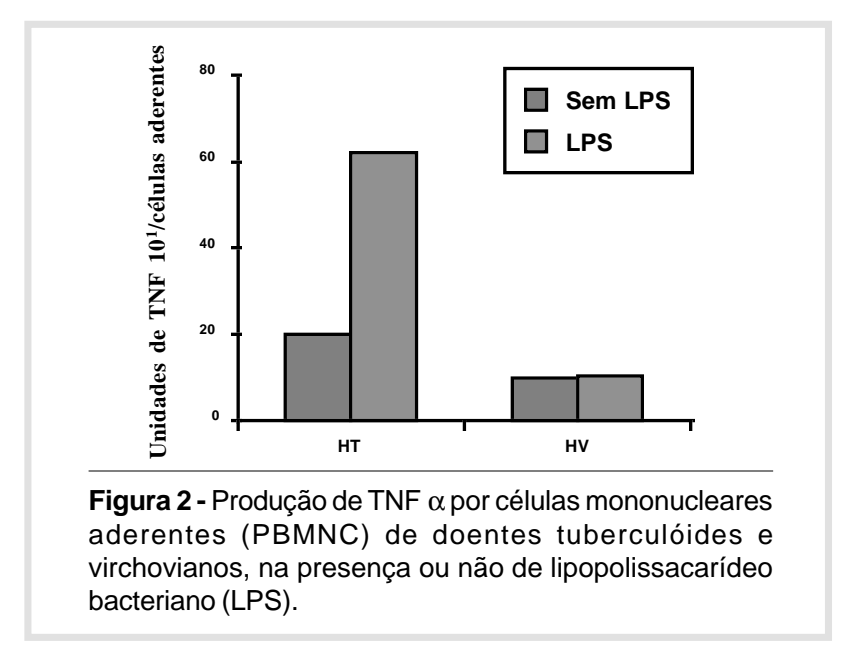

Foi observado, também, que durante o estado reacional, especialmente na reação do tipo eritema nodoso, comum em virchovianos, há aumento da produção de TNF- $\alpha$, associado com elevação dos níveis de proteína-C-reativa (CRP). Isto que sugere que TNF- $\alpha$, citocina inflamatória, pode atuar, estimulando a reação inflamatória aguda, medida pelos altos níveis de CRP, que favorece o aparecimento dos sintomas característicos do surto reacional ${ }^{10}$.

Estes resultados são relevantes na fisiopatologia da hanseníase, pois caracterizaram o TNF- $\alpha$ como a citocina envolvida na defesa, por atividade macrofágica, e na reação inflamatória do eritema nodoso que compromete acentuadamente o estado geral do paciente.

Adicionalmente, podem estar associados à integração da resposta inflamatória aguda, na qual ocorre aumento das citocinas pró-inflamatórias (IL-1, IL-6 e TNF- $\alpha$, principalmente). IL-1 e IL- 6 atuam sobre o hepatócito, estimulando a produção das proteínas da reação inflamatória, entre elas a proteína-C-reativa.

Quando foi investigado o perfil das citocinas IL-1, IL-4 e TNF- $\alpha$, dentro do espectro da hanseníase (Tabela I e Figura 3), observou-se que as concentrações de IL- 1 e TNF- $\alpha$ foram maiores nas formas tuberculóides e dimorfas-tuberculóides, enquanto que as outras formas dimorfas (dimorfa e virchoviana) e virchoviana apresentaram níveis baixos dessas citocinas, correlacionados com altas concentrações de IL- $4^{11}$. 
Tabela I - Níveis de anticorpo anti-PGL1 (Média \pm EPM) e das citocinas IL-1, IL-4, IL-6 e TNF $\alpha$ (medianas) nas diferentes formas clínicas da hanseníase

\begin{tabular}{lrrrrr}
\hline \multicolumn{5}{c}{ Medianas } \\
\hline Classificação & IL-1 & IL-4 & IL-6 & \multicolumn{1}{c}{$\begin{array}{l}\text { TNF- } \alpha \\
(\mathrm{pg} / \mathrm{ml})\end{array}$} & $\begin{array}{c}\text { Anti PGL1 } \\
\text { X EPM }\end{array}$ \\
\hline HT & 44,9 & 0,0 & 12,5 & 66,8 & $21,9 \pm 8,7$ \\
HDT & 22,5 & 2,6 & 12,7 & 40,7 & $32,5 \pm 8,6$ \\
HDV & 3,2 & 60,6 & 6,9 & 0,0 & $81,5 \pm 15,7$ \\
HV & 0,0 & 63,4 & 4,3 & 0,0 & $104,9 \pm 10,4$ \\
ENL & 27,8 & 59,9 & 13,9 & 101,5 & $108,5 \pm 10,3$ \\
\hline
\end{tabular}

EPM - Erro padrão da média; HT - Hanseníase tuberculóide; HDT - Hanseníase dimorfa tuberculóide; HDV - Hanseníase dimorfa virchoviana; HV - Hanseníase virchoviana; ENL - Eritema nodoso lepromatoso.

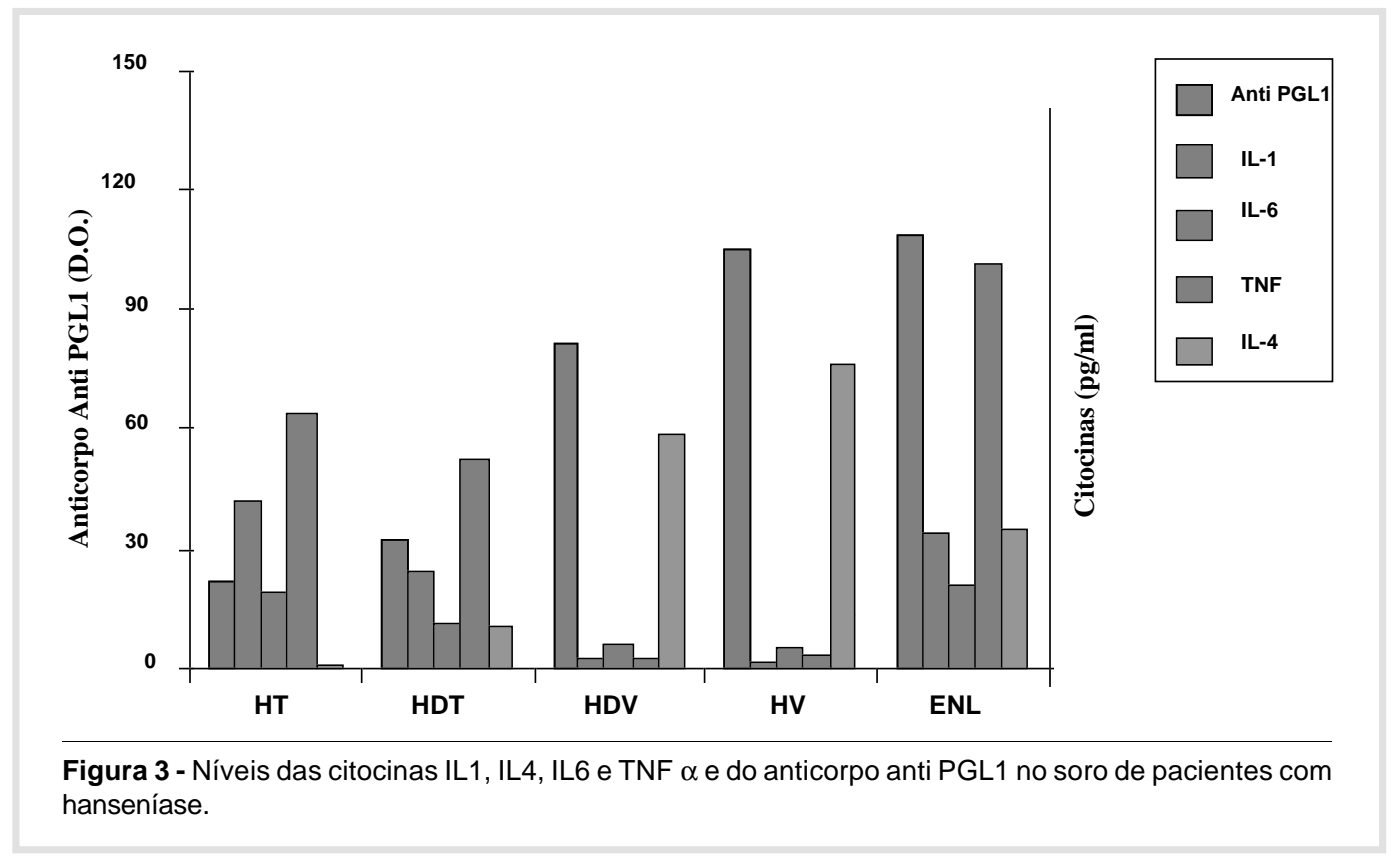

Estes dados sugerem que a produção de IL-4, citocina supressora da atividade macrofágica, está relacionada à depressão da resposta imunológica.

Ainda dentro deste espectro, foi observado que as concentrações do anticorpo anti-PGL1 foram acentuadamente maiores nas formas bacilíferas ${ }^{12}$, estando associadas com aumento significativo de IL-4 e redução de TNF- $\alpha$ e IL- $1^{13}$. Entretanto, durante o surto de eritema nodoso, mesmo com elevados níveis de anti-PGL1, ocorre aumento de TNF- $\alpha$ e redução de IL-4. Adicionalmente, foi observado que o tratamento poliquimioterápico, específico para hanseníase, reduz os níveis de IL-4 e o fluxo bacilar (redução da quantidade de anti-PGL1) com consequiente aumento de TNF- $\alpha^{13}$.
As alterações imunológicas, avaliadas no sangue periférico ou no sobrenadante de culturas, foram correlacionadas com os resultados do estudo imuno-histoquímico, que mostraram que as formas altamente bacilíferas (dimorfa-virchoviana e virchoviana) cursam com elevação acentuada da citocina TGF- $\beta 1$ e de células CD8+ no infiltrado inflamatório. Nas formas de resistência (tuberculóide e dimorfa-tuberculóide), entretanto, foi observado número elevado de células CD4+ e ausência de TGF- $\beta 1^{14}$.

Além disso, em estudo recente, foi observado que, em virchovianos e dimorfos-virchovianos (bacilíferos), a produção de intermediários do nitrogênio avaliada pela enzima NO sintase induzida, está reduzida 
ou ausente ${ }^{15}$, o que pode ser relacionado à presença de TGF- $\beta 1$, células CD8+ e IL-4, no infiltrado inflamatório da lesão cutânea.

Portanto, pode-se concluir que a imunossupressão da hanseníase virchoviana está associada à de- pressão da atividade de macrófagos, estimulação de células $\mathrm{T}$ supressoras $(\mathrm{CD} 8+)$ e à presença das citocinas IL-4 e TGF- $\beta 1$ e, ainda, que esta supressão pode ser atenuada pelo tratamento, ou durante o surto de eritema nodoso.

FOSS NT. Immunological aspects of leprosy. Medicina, Ribeirão Preto, 30: 335-339, july/sept. 1997.

ABSTRACT: The clinical spectrum of leprosy has been associated with immunological alterations induced by the parasite M.leprae on the host. It was observed that lepromatous leprosy present a depressed cellular immune response associated with elevated production of suppressor cytokines (IL-4 and TGF $\beta$ ) and reduced levels of cytokines related with the induction of the immune response (IL-1,TNF $\alpha$ and IFN $\gamma$ ). Furthemore, all these alterations are associated with depressed production of intracellular oxidative elements responsible for the parasite destruction, the reactives of oxigen and nitrogen, the latter evaluated by the enzyme induced NO synthase (iNOS). On the other hand, in tuberculoid leprosy, high levels of inflammatory cytokines and absence of suppressor cytokines have been described.

UNITERMS: Leprosy; immunology.

\section{REFERÊNCIAS BIBLIOGRÁFICAS}

1 - BLOOM BR. Learning from leprosy: a perspective on immunology and the third world. J Immunol 137: 1-10, 1986.

2 - HUNTER SW \& BRENNAN PJ. A novel phenolic glycolipid from Mycobacterium leprae possibly involved in immunogenicity and pathogenicity. J Bacteriol 147: 728-735, 1981.

3 - HUNTER SW; FUJIWARA T \& BRENNAN PJ. Structure and antigenicity of the major specific antigen of Mycobacterium leprae. J Biol Chem 257: 15072-15078, 1982.

4 - HUNTER SW; GAYLORD H \& BRENNAN PJ. Structure and antigenicity of the phosphorylated lipopolysaccharide antigens from the leprosy and tubercle bacilli. J Biol Chem 261: 12345-12351, 1986.

5 - YOUNG DB. Detection of mycobacterial lipids in skin biopsies from leprosy patients. Int J Lepr 49: 198-204, 1981.

6 - HUNTER SW; STEWART C \& BRENNAN PJ. Purification of phenolic glycolipid I from armadillo and human sources. Int $\mathbf{J}$ Lepr 53: 484-486, 1985.

7 - MURRAY HW; SPITALNY GL \& NATHAN CF. Activation of mouse peritoneal macrophages in vitro and in vivo by interferon-gamma. J Immunol 134: 1619-1622, 1985.

8 - CHAN $J$ et al. Killing of virulent Mycobacterium tuberculosis by reactive nitrogen intermediates produced by activated murine macrophages. J Exp Med 175: 1111-1122, 1992.

9 - SILVA CL \& FOSS NT. Tumor necrosis factor in leprosy. J Infect Dis 159: 787-790, 1989.
10 - FOSS NT; OLIVEIRA EB \& SILVA CL. Correlation between TNF production, increase of plasma-C- reactive protein level and suppression of $\mathrm{T}$ lymphocyte response to Concanavalin A during erythema nodosum leprosum. Int J Lepr 61: 218-226, 1993.

11 - ALMEIDA AM. Concentração sérica de citocinas no espectro das formas clínicas da hanseníase. Dissertação de Mestrado, Faculdade de Medicina de Ribeirão Preto, da USP. Ribeirão Preto, 1996.

12 - FOSS NT; CALLERA F \& ALBERTO FL. Anti-PGL-1 levels in leprosy patients and their contacts. Braz $\mathbf{J}$ Med Biol Res 26: 43-51, 1993.

13 - BONATO VLD. Correlação dos anticorpos anti PGL-1 com o índice baciloscópico, a reação de Mitsuda, o tratamento poliquimioterápico e as interleucinas nas diferentes formas da hanseníase. Dissertação de Mestrado, Faculdade de Medicina de Ribeirão Preto da USP. Ribeirão Preto, 1995.

14 - OLIVEIRA MSA. Correlação entre a resposta imunocelular periférica e características imunohistoquímicas das lesões cutâneas nas formas polares da hanseníase. Tese de Doutorado. Faculdade de Medicina de Ribeirão Preto da USP. Ribeirão Preto, 1996.

15 - FIGUEIREDO F et al. Detection of nitric oxide (NO) in dermal lesion of patients with leprosy, comunicação pessoal.

Recebido para publicação em 02/07/97

Aprovado para publicação em 30/07/97 